This last diet gave the most unfavourable results as for body composition. The results of all the other treatments were less good as those obtained with the control diet (body composition and food conversion ratio) except in the case where the ration contained ro p. Ioo wheat bran and I 8 p. roo CP : same food conversion ratio, batter carcasses.

During the second trial, the author usad all the precited diets, except the last one. The food distribution was limited at $2, \mathrm{I} \mathrm{kg}$ per day.

The body composition was almost identical for a!l the animals, but the food conversion ratio was definitely in favour of the controls (3.01 compared with 3.21-3.58). The advantage of wheat bran at the lowest level was only partially recovered (FCR $=3.21$; other treatments : 3.46 - 3.58).

The author concludes that the addition of callulose to a " maize + soybean "diet is without any use ; a moderate level of wheat bran being however justified during the " pre-fattening " period.

\title{
UTILISATION DE L'ENSILAGE DE MAÏS GRAIN HUMIDE PAR LE PORC EN CROISSANGE ET FINITION
}

\author{
J. P. KERMOAL, M. L'AOT et J. P. LOSSEC \\ Maison de l'Élevage du Finistère, \\ $29 S$ - Quimper
}

\section{RÉSUMÉ}

3 régimes alimentaires à base d'orge, de maïs grain sec et d'ensilage de maïs grain humide broyé ont été comparés sur des porcs en croissance et finition (entre 35 et $100 \mathrm{~kg}$ ).

Les 3 régimes étaient équilibrés par un apport de tourteau de soja et d'un composé minéral vitaminisé.

Les porcs étaient alimentés suivant un plan de rationnement jusqu'à $60 \mathrm{~kg}$, ensuite la ration était constante. Les rations étaient calculées de manière à apporter la même quantité d'énergie digestible et de matières azotées totales pour les trois régimes.

Les deux régimes maïs se sont montrés identiques pour tous les critères étudiés (croissance, indice de consommation, composition corporelle).

Le régime orge a donné une croissance égale aux régimes mais, mais avec un indice de consommation $(\mathrm{kg} \mathrm{M} / \mathrm{kg}$ ) significativement plus élevé.

Les porcss nourris au régime orge avaient tendance à être plus maigres que ceux nourris aux régimes maïs.

\section{SUMMARY \\ UTILIZATION OF HUMID MAIZE GRAIN SILAGES \\ IN THE GROWING-FINISHING PIG}

3 food diets containing barley, dry maize grain and silages of grinded humid maize grain were compared in growing-finishing pigs (between 35 and roo $\mathrm{kg}$ ).

The 3 diets were balanced by a supply of soybean meal and a vitaminized mineral component. 
The pigs were fed according to a schedule of rationing until $60 \mathrm{~kg}$, and then received a constant ration which was calculated so that the diets contained the same amount of digestible energy and total crude protein.

The two maize diets gave identical results as far as growth, food conversion ratio and body composition are concerned.

The barley diet gave the same growth in the animals as that obtained with the maize diets, but the food conversion ratio $(\mathrm{kg} \mathrm{DM} / \mathrm{kg}$ ) was significantly higher.

The barley fed pigs had a tendency to be leaner compared with those receiving the maize diets.

\title{
VALEUR ALIMENTAIRE DU MAÏS ENSILE \\ A DIFFÉnENTS STADES DE MATURITÉ pour la truie, le porcelet et le porc en choissance-finition
}

\author{
C. FÉVRIER, A. AUMAITRE et E. SALMON-LEGAGNEUR
}

Station de Recherches sur l'Élevage des Porcs,

Centre national de Recherches zootechniques, I. N. R. A., 78 - Jouy-en-Josas

\section{RÉSUMÉ}

Du maïs $I . N . R . A .258$ a été récolté et conservé suivant différentes méthodes : en grain séché et récolté à maturité GSM ; en grain ensilé à 35 p. Ioo d'humidité, récolté à maturité, GEM ; en grain ensilé à $4^{\circ}$ p. Ioo d'humidité, récolté immature, GEI ; en épi entier ensilé à 56 p. Ioo d'humidité, récolté immature, EEI. La valeur alimentaire de régimes à base de ces différents produits a été étudiée par des expériences en lots et en bilans sur des truies (GSM, GEM et EEI), des porcelets entre 5 et 9 semaines (GSM et GEI), des porcs en croissance-finition (GSM, GEM, GEI, EEI).

Chez la truie, comme chez le porc en croissance, l'utilisation digestive de l'énergie et de l'azote du régime EEI est très significativement inférieure à celle des autres régimes. Pour le grain, on n'observe pas de différence en ce qui concerne la digestibilité de l'azote ; par cortre, celle de l'énergie est significativement plus faible pour GEM que pour GSM, pour la truie comme pour le porc. Ainsi pour la truie la valeur en énergie digestible est, en $\mathrm{kcal} / \mathrm{kg}$ de matière sèche : GSM 3800 ; GEM 3580 ; EEI 3 390, et pour le por: : GSM 3980 ; GEI 3930 ; GEM $3790 ;$ EEI 3470. Pour le porcelet la différence de digestibilité de l'énergie entre GSM et GEI est également nulle mais, par contre, la digestibilité de l'azote est plus élevée pour GEI que pour GSM, notamment dans les premières semaines. Les résultats zootechniques confirment ceux de l'étude de digestibilité. Ainsi, chez la truie, bien que les portées à la naissance ne soient pas différentes entre les lots, le poids des porcelets à 35 jours est plus élevé po:ır GSM que pour GEM ou EEI et pour ce dernier lot le bilan du gain de poids gestation-lactation est fortement négatif. Les porcelets recevant GEI ont consommé un peu plus d'aliment que les témoins GSM, mais la croissance a été identique. Pour les porcs recevant EEI, la vitesse de croissance et l'efficacité alimentaire ont été significativement plus faibles que pour ceux recevant le maïs grain. Sur l'ensemble de la croissance, les résultats ont été identiques pour les trois autres lots, mais la composition de la carcasse était signi . ficativement plus maigre pour GEM, ce qui traduit une valeur énergétique plus iaible pour ce 